\title{
1 Wave interaction with a perforated circular breakwater of non-uniform porosity
}

\author{
Longbin Tao and Hao Song \\ Griffith School of Engineering, Gold Coast campus, Griffith University, QLD4222, Australia \\ (l.tao@griffith.edu.au) \\ Subrata Chakrabarti \\ Offshore Structure Analysis, Inc., Plainfield, IL60544-7096, USA \\ May 26, 2008
}

Abstract. In this paper, wave interaction with a porous cylindrical breakwater is studied analytically by linear potential wave theory. The breakwater is assumed to have a thin skin, is bottom-mounted and surface-piercing. The porosity of the breakwater is uniform vertically but varies in the circumference direction. This allows the choice of a partially impermeable wall or a vertical slot in the breakwater. Three different basic configurations of the breakwater are investigated, namely, (1) uniformly porous cylinder; (2) porous cylinder with partial impermeable wall; and (3) porous cylinder with an opening. The performance of these types of breakwaters is studied versus wave parameters and breakwater configurations including angle and position of opening or partial impermeable wall as well as porosity. Parametric studies with regard to the wave amplification factor, wave forces, and elevation contours are made. The results should be found useful in the design of coastal and offshore structures.

Keywords: short-crested wave, wave diffraction, circular breakwater, porous structure

Porous breakwaters are often constructed to reduce the wave impact on coastal and offshore structures. They can also reduce resonance more effectively than an impermeable breakwater [1]. Since the early work of Jarlan [2], wave interaction with a porous breakwater has attracted the attention of many coastal and offshore researchers. In one instance among many, Dalrymple et al. [3] studied the reflection and transmission of a wave train at an oblique angle of incidence by an infinitely long porous breakwater. Subsequently, Huang and Chao [4] reported the inertial effect of the porous breakwater based on Biot's theory of poroelasticity.

Following the porous wavemaker theory of Chwang [5] and subsequent works, investigations have primarily been concentrated on the hydrodynamic effects of a porous structure on the incoming wave trains, or wave impact on porous structures as a breakwater in a harbour (e.g., [1, 6, 7]). In most cases, Darcy's law for a homogeneous porous medium has been applied. Yu and Chwang [6] investigated the resonance in a harbour with porous breakwaters subjected to an arbitrary wave angle followed by an extensive study on the

(c) 2008 Kluwer Academic Publishers. Printed in the Netherlands. 
wave transmission characteristics past a porous structure [7]. They also investigated the behaviour of waves within the porous medium. It was found that there is an optimum thickness for a porous structure beyond which any further increase in the thickness may not lead to an appreciable improvement in reducing its transmission and reflection characteristics. Wang and Ren [8] studied the performance of a flexible porous breakwater, and found that hydrodynamic forces on the interior cylinder as well as wave amplitudes around the windward side of the interior cylinder are reduced when compared to the case of a direct wave impact on the interior cylinder. More related works can be found in the review article of Chwang and Chan [1].

The aforementioned studies on the interaction of ocean surface waves with a vertical porous breakwater have generally been two-dimensional. In reality, however, the ocean waves are more complex, and better described by three-dimensional (3D) short-crested waves. They also commonly arise, for example, from the oblique interaction of two travelling plane waves or intersecting swell waves, or from the reflection of waves at non-normal incidence off a vertical seawall, as well as from the diffraction about the surface boundaries of a structure of finite length. These multi-directional, multi-component waves are of paramount importance in coastal and offshore engineering design. In contrast to plane waves propagating in a single direction, and the standing waves fluctuating vertically in a confined region, short-crested waves can be doubly periodic in two horizontal directions, one in the direction of propagation and the other normal to it [9].

Theoretical analysis on short-crested wave interaction with a vertical cylinder can be found in [10-12]. Zhu [10] presented an analytic solution to the diffraction problem for a solid circular cylinder in short-crested waves using linear potential wave theory and found that the pressure distribution and wave run-up on the cylinder were quite different from those of plane incident waves. Their patterns become very complex as $k a$ (i.e., total incident wave number $k$ times cylinder radius $a$ ) becomes large. The hydrodynamic forces on the cylinder become smaller as the short-crestedness of the incident waves increases. Subsequently, Zhu and Moule [11] observed that the hydrodynamic force induced by short-crested waves varies with the phase angle perpendicular to the direction of wave propagation. Later, Zhu and Satravaha [12] extended the analytical solution for the velocity potential to second-order.

Although efforts have been made on wave interaction with porous cylinders and breakwaters, there is no relevant work on the wave interaction with a perforated cylindrical breakwater having variable porosity and opening. In this paper, analytical solutions are derived to study this problem in a quantitative manner. Detailed numerical results are presented over a broad range of incident short-crested wave parameters as well as structural configurations including the porosity of the breakwater and the angle and position of the impermeable wall and opening. In particular, their effects on wave amplifica- 
tion factors, wave forces, and wave elevation contours near the structure are discussed.

\section{Theoretical Consideration}

\subsection{PROBlem DESCRIPTION}

It is worth noting that theoretical derivation can be made on 2D plane waves, and the linear solutions of 3D short-crested waves can be obtained by linear superposition of two plane waves. We intend to extend this study to include nonlinear effects. Therefore, in this section, the mathematical formulae are derived for a general case of interaction of 3D short-crested waves with a porous cylindrical breakwater of variable porosity. Note that the solutions for the 2D limiting cases, i.e., a plane incident wave and a standing wave, can be instantly recovered from it by letting $k_{y}=0$ and $k_{x}=0$ ( $k_{x}=$ wave number in $x$ direction, $k_{y}=$ wave number in $y$ direction) respectively.

Consider a monochromatic short-crested wave train propagating in the direction of the positive $x$ axis. A perforated cylindrical breakwater extends from the sea bottom to above the free surface of the ocean along $z$ axis. The origin is placed at the centre of the cylindrical breakwater on the mean water surface (see Fig. 1). A partially impermeable wall or opening is located at $\theta \in\left(\varepsilon_{1}, \varepsilon_{2}\right)$ in cylindrical coordinates $(r, \theta, z)$. The whole fluid region is divided into two regions - the region inside the breakwater, $\Omega_{1}$ and the region outside the breakwater, $\Omega_{2}$. The following notation are used in the paper: $\Phi_{j}=$ total velocity potential, $\Phi_{j}^{I}=$ velocity potential of incident wave, $\Phi_{j}^{S}=$ velocity potential of scattered wave, $k=$ total wave number, $\omega=$ wave frequency, $h=$ water depth, $A=$ amplitude of incident wave, $a=$ radius of the cylindrical breakwater, $t=$ time, $\rho=$ mass density of water, and $g=$ gravitational acceleration. The subscripts $j(j=1,2)$ denote the physical parameters in the region $\Omega_{j}(j=1,2)$.

Assume that the fluid is inviscid and incompressible, and the flow is irrotational. Then the fluid flow can be described by a velocity potential $\Phi_{j}$, which satisfies the Laplace equation

$$
\nabla^{2} \Phi_{j}=0 \quad \text { in } \quad \Omega_{j},
$$

subject to the combined linearised free surface boundary condition

$$
\Phi_{j, t t}+g \Phi_{j, z}=0 \quad \text { at } \quad z=0,
$$

and the bottom condition

$$
\Phi_{j, z}=0 \quad \text { at } \quad z=-h,
$$




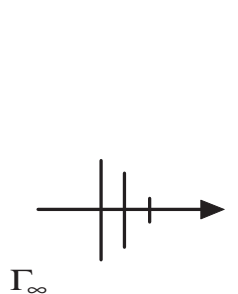

$$
\Gamma_{\infty}
$$

$$
\Omega_{2}
$$
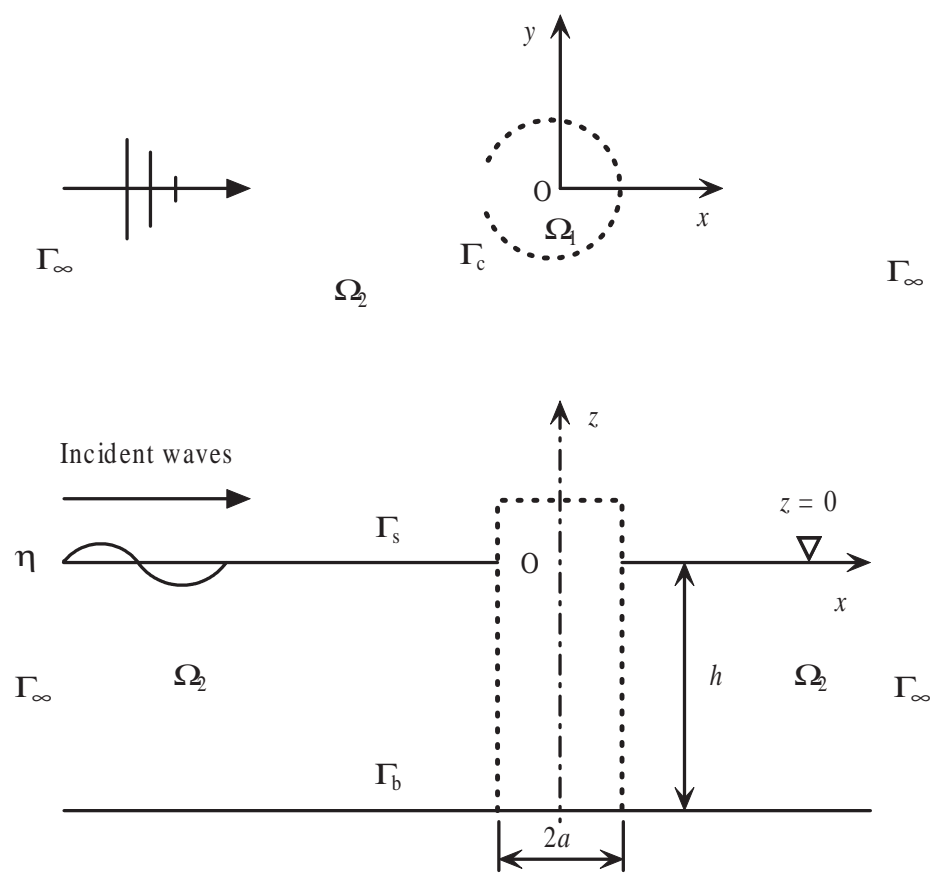

Figure 1. Definition sketch of short-crested waves on a porous cylindrical breakwater.

where the comma in the subscript designates partial derivative with respect to the variable following it.

The total velocity potential in region $\Omega_{2}$ can be expressed by the summation of the incident and scattered wave velocity potentials

$$
\Phi_{2}=\Phi_{2}^{I}+\Phi_{2}^{S} \quad \text { in } \quad \Omega_{2},
$$

where $\Phi_{2}^{I}$ and $\Phi_{2}^{S}$ also satisfy (1) - (3).

The velocity potential of the linear short-crested incident wave [13] travelling principally in the positive $x$ direction is given by the real part of

$$
\Phi_{2}^{I}=-\frac{i g A}{\omega} f(z, h) e^{i\left(k_{x} x-\omega t\right)} \cos \left(k_{y} y\right) \quad \text { in } \quad \Omega_{2},
$$

where $k^{2}=k_{x}^{2}+k_{y}^{2}$, and

$$
f(z, h)=\frac{\cosh k(z+h)}{\cosh k h} .
$$

The term $f(z, h)$ leads to the sea bottom condition being automatically satisfied, while the linearised free surface boundary condition is satisfied using the following dispersion relationship

$$
\omega^{2}=g k \tanh k h .
$$


Assuming that the fluid flow passing through the perforated breakwater as a porous boundary obeys Darcy's law [14], the boundary condition on perforated breakwater can be expressed as [5]

$$
\begin{gathered}
\Phi_{1, r}=\Phi_{2, r}=i G(\theta) k\left(\Phi_{1}-\Phi_{2}\right) \quad \text { on } \quad r=a, \\
\Phi_{2, r}^{S}=i G(\theta) k\left(\Phi_{1}-\Phi_{2}^{S}-\Phi_{2}^{I}\right)-\Phi_{2, r}^{I} \quad \text { on } \quad r=a,
\end{gathered}
$$

where $r$ is the radial axis, $i=\sqrt{-1}, G(\theta)=\frac{\rho \omega d(\theta)}{\mu}$ is a measure of the porosity, $\mu$ is the coefficient of dynamic viscosity, $d(\theta)$ is a material constant having the dimension of length. The porous effect parameter $G$ is a dominant parameter in the present study. Its value depends on the geometrical parameters of the permeable wall and wave factors [15]. The geometrical parameters of a permeable wall consist mainly of geometrical porosity, plate thickness and porous shape. In engineering practices, the geometrical porosity is about $20 \%$ and can reach as high as $60 \%$ or higher in some circumstances. Several porous shapes are common in coastal or offshore structures, including slit, screen and circular or rectangular holes. Detailed method of estimate of $G$ could be found in [15]. In addition, the scattered potential satisfies the Sommerfeld radiation condition at infinity as follows:

$$
\lim _{k r \rightarrow \infty}(k r)^{1 / 2}\left(\Phi_{2, r}^{S}-i k \Phi_{2}^{S}\right)=0 \quad \text { in } \quad \Omega_{2} .
$$

Therefore, the scattered wave velocity potential $\Phi_{2}^{S}$ in $\Omega_{2}$ is governed by the Laplace equation (1) with the boundary conditions (2) and (3), the boundary condition at the interface of fluid and breakwater at $r=a(8)$ and (9), and the radiation condition (10).

The velocity potential $\Phi_{1}$ in the interior domain $\Omega_{1}$ is governed by the Laplace equation (1) with the boundary conditions (2) and (3), and the boundary conditions at the interface of fluid and breakwater at $r=a$ :

$$
\Phi_{1, r}=i G(\theta) k\left(\Phi_{1}-\Phi_{2}^{S}-\Phi_{2}^{I}\right) \quad \text { on } \quad r=a .
$$

These constitute two sets of the governing equation and corresponding boundary conditions for the diffraction of short-crested waves by a vertical perforated cylindrical breakwater with nonuniform porosity, corresponding to boundary-value problems in a bounded domain and an unbounded domain respectively. After obtaining $\Phi_{2}^{S}, \Phi_{2}$ and $\Phi_{1}$ by solving the above boundaryvalue problems, all the physical quantities including the fluid particle velocity, free surface elevation and the dynamic pressure can be calculated respectively from

$$
\mathbf{v}_{j}=\nabla \Phi_{j}
$$




$$
\begin{gathered}
\eta_{j}=\left.\frac{i \omega}{g} \Phi_{j}\right|_{z=0, t=0}, \\
p_{j}=-\rho \Phi_{j, t} .
\end{gathered}
$$

$\Phi_{2}^{I}=-\frac{i g A}{\omega} f(z, h) e^{-i \omega t}\left[\sum_{m=0}^{+\infty} \varepsilon_{m} i^{m} J_{m}\left(k_{x} r\right) \cos (m \theta)\right]\left[\sum_{n=0}^{+\infty} \varepsilon_{n} J_{2 n}\left(k_{y} r\right) \cos (2 n \theta)\right]$,

157

where

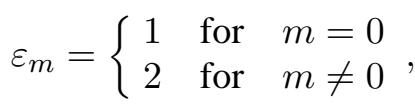

$$
\begin{aligned}
\psi_{l}\left(k_{x} r, k_{y} r\right) & =\frac{1}{2}\left\{\sum_{n=\max \{0,\lceil(l-M) / 2\rceil\}}^{\min \{N,\lfloor l / 2\rfloor\}} \varepsilon_{l-2 n} \varepsilon_{n} i^{l-2 n} J_{l-2 n}\left(k_{x} r\right) J_{2 n}\left(k_{y} r\right)\right. \\
& +\sum_{n=0}^{\min \{N,\lfloor(M-l) / 2\rfloor\}} \varepsilon_{l+2 n} \varepsilon_{n} i^{l+2 n} J_{l+2 n}\left(k_{x} r\right) J_{2 n}\left(k_{y} r\right) \\
& \left.+\sum_{n=\lceil l / 2\rceil}^{\min \{N,\lfloor(M+l) / 2\rfloor\}} \varepsilon_{2 n-l} \varepsilon_{n} i^{2 n-l} J_{2 n-l}\left(k_{x} r\right) J_{2 n}\left(k_{y} r\right)\right\},(19)
\end{aligned}
$$




$$
\Phi_{2}^{S}=-\frac{i g A}{\omega} f(z, h) e^{-i \omega t}\left\{\sum_{l=0}^{L} A_{l}^{1} \cos (l \theta) H_{l}(k r)+\sum_{l=1}^{L} A_{l}^{2} \sin (l \theta) H_{l}(k r)\right\},
$$

in which $\lceil\quad\rceil$ is a function giving the greatest integer less than or equal to its argument and $\lfloor\quad\rfloor$ is a function returning the smallest integer greater than or equal to its argument.

According to [6] and [10], the evanescent waves do not exist in the absence of related boundary conditions. The solution of the scattered velocity potential in region $\Omega_{2}$ can be constructed by the following expression

which satisfies the Laplace equation (1), boundary conditions (2) and (3), and the Sommerfeld radiation condition (10) for all $A_{l}^{1}$ and $A_{l}^{2}$, where $H_{l}$ is the Hankel functions of the first kind, and $A_{l}^{1}$ and $A_{l}^{2}$ are unknown complex coefficients.

Similarly, the solution of the velocity potential in the interior region $\Omega_{1}$ can be constructed as

$$
\Phi_{1}=-\frac{i g A}{\omega} f(z, h) e^{-i \omega t}\left\{\sum_{l=0}^{L} B_{l}^{1} \cos (l \theta) J_{l}(k r)+\sum_{l=1}^{L} B_{l}^{2} \sin (l \theta) J_{l}(k r)\right\},
$$

where $B_{l}^{1}$ and $B_{l}^{2}$ are unknown complex coefficients.

Substituting (18), (20) and (21) into the body boundary conditions (8) and (11), and noting the orthogonality property of the trigonometric functions, we have

$$
\begin{gathered}
B_{l}^{1} J_{l}^{\prime}(k a)=\psi_{l}^{\prime}\left(k_{x} a, k_{y} a\right) / k+A_{l}^{1} H_{l}^{\prime}(k a) \\
B_{l}^{2} J_{l}^{\prime}(k a)=A_{l}^{2} H_{l}^{\prime}(k a) \\
\sum_{l=0}^{L}\left[B_{l}^{1} J_{l}(k a)-A_{l}^{1} H_{l}(k a)-\psi_{l}\left(k_{x} a, k_{y} a\right)\right] \cos (l \theta) \\
+\sum_{l=1}^{L}\left[B_{l}^{2} J_{l}(k a)-A_{l}^{2} H_{l}(k a)\right] \sin (l \theta) \\
=\frac{1}{i G(\theta)}\left\{\sum_{l=0}^{L} B_{l}^{1} J_{l}^{\prime}(k a) \cos (l \theta)+\sum_{l=1}^{L} B_{l}^{2} J_{l}^{\prime}(k a) \sin (l \theta)\right\} \quad(G \neq 0),
\end{gathered}
$$

where the prime denotes the derivative with respect to $r$.

It should be noted that (24) is not appropriate when $G=0$. However, if a very small value (e.g. $1 e^{-12}$ ) is assigned to $G(\theta)$, representing the case of impermeable wall, (24) still applies and leads to highly accurate results. 
From (22) and (23), we have

$$
\begin{gathered}
B_{l}^{1}=\frac{\psi_{l}^{\prime}\left(k_{x} a, k_{y} a\right)+k H_{l}^{\prime}(k a) A_{l}^{1}}{k J_{l}^{\prime}(k a)}, \quad l=0,1,2, \ldots, L, \\
B_{l}^{2}=\frac{H_{l}^{\prime}(k a)}{J_{l}^{\prime}(k a)} A_{l}^{2}, \quad l=1,2, \ldots, L .
\end{gathered}
$$

in which

$$
\begin{gathered}
\mathbf{A}=\left[A_{0}^{1}, A_{1}^{1}, \cdots, A_{L}^{1}, A_{1}^{2}, \cdots, A_{L}^{2}\right]^{T}, \\
\mathbf{B}=\left[B_{0}^{1}, B_{1}^{1}, \cdots, B_{L}^{1}, B_{1}^{2}, \cdots, B_{L}^{2}\right]^{T} \\
\mathbf{C}=-\mathbf{Q \Psi} \\
\mathbf{D}=-\mathbf{Q H} \\
\mathbf{E}=\mathbf{Q J}+i \mathbf{S} \mathbf{J}^{\prime} \\
\mathbf{Q}=\operatorname{diag}[2 \pi, \pi, \pi, \cdots, \pi], \\
\mathbf{H}=\operatorname{diag}\left[H_{0}(k a), H_{1}(k a), \cdots, H_{L}(k a), H_{1}(k a), \cdots, H_{L}(k a)\right] \\
\mathbf{\Psi}=\operatorname{diag}\left[\psi_{0}\left(k_{x} a, k_{y} a\right), \psi_{1}\left(k_{x} a, k_{y} a\right), \cdots, \psi_{L}\left(k_{x} a, k_{y} a\right), 0, \cdots, 0\right], \\
\mathbf{J}^{\prime}=\operatorname{diag}\left[J_{0}^{\prime}(k a), J_{1}^{\prime}(k a), \cdots, J_{L}^{\prime}(k a), J_{1}^{\prime}(k a), \cdots, J_{L}^{\prime}(k a)\right]
\end{gathered}
$$


$\mathbf{S}_{j l}=\left\{\begin{array}{l}\int_{0}^{2 \pi} \frac{1}{G(\theta)} \cos (j \theta) \cos (l \theta) d \theta \quad 0 \leq j \leq L, 0 \leq l \leq L, \\ \int_{0}^{2 \pi} \frac{1}{G(\theta)} \cos (j \theta) \sin (l-L) \theta d \theta \quad 0 \leq j \leq L, L+1 \leq l \leq 2 L+1, \\ \int_{0}^{2 \pi} \frac{1}{G(\theta)} \sin (j-L) \theta \cos (l \theta) d \theta \quad L+1 \leq j \leq 2 L+1,0 \leq l \leq L, \\ \int_{0}^{2 \pi} \frac{1}{G(\theta)} \sin (j-L) \theta \sin (l-L) \theta d \theta \quad L+1 \leq j \leq 2 L+1, L+1 \leq l \leq 2 L+1,\end{array}\right.$

where "diag" denotes a diagonal matrix with the elements in the square brackets on the main diagonal.

(25), (26) and (27) constitute a set of linear equations for $A_{l}^{1}, A_{l}^{2}, B_{l}^{1}$, and $B_{l}^{2}$. Once the values of these coefficients are obtained, all the physical quantities can be calculated accordingly.

\subsection{Physical QUANTITIES}

The elevations in the interior and exterior regions are

$$
\begin{gathered}
\eta_{1}=A\left\{\sum_{l=0}^{L} B_{l}^{1} J_{l}(k r) \cos (l \theta)+\sum_{l=1}^{L} B_{l}^{2} J_{l}(k r) \sin (l \theta)\right\} \\
\eta_{2}=A\left\{\sum_{l=0}^{L}\left[\psi_{l}\left(k_{x} r, k_{y} r\right)+A_{l}^{1} H_{l}(k r)\right] \cos (l \theta)+\sum_{l=1}^{L} A_{l}^{2} H_{l}(k r) \sin (l \theta)\right\} .
\end{gathered}
$$

The pressures on the boundary (interior and exterior) are

$$
p_{1}=\rho g A f(z, h) e^{-i \omega t}\left\{\sum_{l=0}^{L} B_{l}^{1} J_{l}(k a) \cos (l \theta)+\sum_{l=1}^{L} B_{l}^{2} J_{l}(k a) \sin (l \theta)\right\}
$$

$p_{2}=\rho g A f(z, h) e^{-i \omega t}\left\{\sum_{l=0}^{L}\left[\psi_{l}\left(k_{x} a, k_{y} a\right)+A_{l}^{1} H_{l}(k a)\right] \cos (l \theta)+\sum_{l=1}^{L} A_{l}^{2} H_{l}(k a) \sin (l \theta)\right\}$.

The total force per unit length in the direction of $s(s=x, y)$ is

$$
\frac{d F_{s}}{d z}=a\left[\int_{0}^{2 \pi}\left(p_{1}-p_{2}\right) \cdot \varphi_{s} d \theta\right]=P_{s}\left(k_{x}, k_{y}, k, a\right) \cdot \rho g a A \cdot f(z, h) e^{-i \omega t},
$$

where the function $P_{s}\left(k_{x}, k_{y}, k, a\right)$ is a nondimensional parameter of $\frac{d F_{s}}{d z}$ without the constant term $\rho g a A \cdot f(z, h) e^{-i \omega t}$, and 


$$
\varphi_{x}=\cos (\theta), \quad \varphi_{y}=\sin (\theta) .
$$

By the orthogonality of the trigonometric functions, only the term $l=1$ in the series (41) and (42) remains, so that the function $P_{s}\left(k_{x}, k_{y}, k, a\right)$ can be expressed explicitly as

$$
\begin{aligned}
& P_{x}\left(k_{x}, k_{y}, k, a\right)=\pi \cdot\left[B_{1}^{1} J_{1}(k a)-\psi_{l}\left(k_{x} a, k_{y} a\right)-A_{1}^{1} H_{1}(k a)\right], \\
& P_{y}\left(k_{x}, k_{y}, k, a\right)=\pi \cdot\left[B_{1}^{2} J_{1}(k a)-A_{1}^{2} H_{1}(k a)\right] .
\end{aligned}
$$

The function $P_{s}\left(k_{x}, k_{y}, k, a\right)$ determines the first-order total force in $s$ ( $s=x, y$ ) direction on the perforated cylindrical structure, $F_{s}$, which can be obtained by integrating (43) with respect to $z$,

$$
F_{s}=\int_{-h}^{0} \frac{d F_{s}}{d z} d z=P_{s}\left(k_{x}, k_{y}, k, a\right) \cdot \rho g h a A e^{-i \omega t} \cdot \tanh (k h) / k h .
$$

The total moments about an axis parallel to the $y$ and $x$ axis passing through the bottom of the cylindrical structure respectively are

$$
\begin{aligned}
& M_{y}=\int_{-h}^{0}(z+h) \frac{d F_{x}}{d z} d z=P_{x}\left(k_{x}, k_{y}, k, a\right) \rho g h^{2} a A e^{-i \omega t} Z(k h), \\
& M_{x}=-\int_{-h}^{0}(z+h) \frac{d F_{y}}{d z} d z=-P_{y}\left(k_{x}, k_{y}, k, a\right) \rho g h^{2} a A e^{-i \omega t} Z(k h),
\end{aligned}
$$

where

$$
Z(k h)=[k h \tanh (k h)+\operatorname{sech}(\mathrm{kh})-1] /(\mathrm{kh})^{2} .
$$

It is noted from (47) - (49) that only the function $P_{s}\left(k_{x}, k_{y}, k, a\right)$ needs to be determined in order to derive all the subsequent results.

\subsection{LIMITING CASE}

For uniform porous cylinder, i.e. $G(\theta)=G_{0}$, matrix $\mathbf{S}$ becomes a diagonal matrix and the solution can be expressed explicitly as

$$
\begin{aligned}
A_{l}^{1} & =-\frac{i \pi k a G_{0}\left[\psi_{l} J_{l}^{\prime}(k a)-\varphi_{l}^{\prime} J_{l}(k a) / k\right]+\pi a \varphi_{l}^{\prime} J_{l}^{\prime}(k a)}{2 G_{0}+\pi k a J_{l}^{\prime}(k a) H_{l}^{\prime}(k a)}, \\
B_{l}^{1} & =\frac{-i \pi k a G_{0}\left[\psi_{l} H_{l}^{\prime}(k a)-\varphi_{l}^{\prime} H_{l}(k a) / k\right]}{2 G_{0}+\pi k a J_{l}^{\prime}(k a) H_{l}^{\prime}(k a)}, \\
A_{l}^{2} & =B_{l}^{2}=0 .
\end{aligned}
$$


Fig. 2 shows the variations of wave amplification factor $(|\eta| / A)$ at the origin $r=0$ (left) and nondimensional wave forces on the breakwater (right) $v s$. $k a$, where Figs. 2(a) and 2(d) correspond to the case of a breakwater with a uniform porosity $\left(G_{0}=1\right)$, and Figs. 2(b \& e) and 2(c \& f) correspond to the cases of a breakwater with a partial solid wall and a partial opening respectively at $175^{\circ}<\theta<185^{\circ}$ with the balance of the porosity remaining at $G_{0}=1$. Cases comprising of five different wave spread angles at $\beta=0$, $\pi / 8, \pi / 4,3 \pi / 8$, and $\pi / 2$ (where $\beta=\arctan \left(k_{y} / k_{x}\right)$ ) are calculated and the results are plotted. As can be seen in Fig. 2(a), all the curves representing wave amplification factors of different wave spread angles coincide with one another. This is a clear indication that the wave amplification factor at origin is independent of the wave spread angle $\beta$ for breakwaters with a uniform porosity. The wave amplification factor at origin is seen to decrease monotonically from 1 to approximately half as $k a$ increases up to about 2.2, and then increase monotonically to about 1 before $k a$ reaches around 3.8 and afterwards fluctuate again. As shown in Fig. 2(b), the variation of wave amplification factor for a breakwater with a partial solid wall is very similar to that of a breakwater with a uniform porosity. However, waves of different $\beta$ values result in slightly different amplification factors. It is seen that a standing wave $(\beta=\pi / 2)$ tends to result in the highest amplification factor whilst the incident short-crested wave with $k_{x}=k_{y}$ produces the lowest amplification factor for a large range of $k a$. As indicated in Fig. 2(c), the variation of amplification factor for the breakwater with a partial opening is similar to that for the breakwater with a uniform porosity at large $k a$. A distinct feature of the variation of amplification factor is that an additional peak is clearly observed for each short-crestedness at around $k a=0.2$, and the maximum amplification factor at origin is about 1.15. Furthermore, as one would expect, a plane wave is seen to result in the highest amplification factor, while a standing wave tends to result in its lowest value for $k a \leq 3$.

For a breakwater with a uniform porosity, the nondimensional wave force in the direction of wave propagation (Figs. 2(d)-2(f)), decreases as the shortcrestedness increases. In fact, the wave force becomes zero when the shortcrestedness arrives at its maximum value (i.e., standing waves), since the configuration is symmetric about the $y$-axis. Peaks and troughs occur at approximately the same $k a$ value for different short-crestedness. However, for the breakwater with a partial solid wall (Fig. 2(e)) or an opening (Fig. 2(f)), the wave forces induced by a standing wave are no longer zero, since now the configuration is nonsymmetric about the $y$-axis and the peaks and troughs for different short-crestedness tend to occur at slightly different values of $k a$. More specifically, the peaks and troughs occur at larger $k a$ for the partial solid wall, while they occur at smaller values of $k a$ for the opening. Due to 
the asymmetry in the configuration, the breakwater with a partial solid wall yields the largest wave force and the one with a partial opening gives the smallest wave force, except for the case of standing incident wave.

Fig. 3 shows the influence of the porosity on the wave amplification factor at the origin $r=0$ (left) and wave forces on the breakwater (right) for a breakwater with uniform porosity $G_{0}$, a partial solid wall and a partial opening at $175^{\circ}<\theta<185^{\circ}$ with porosity of the remaining part $G_{0}$. As can be seen from Figs. 3(a)-3(c), wave spreading angles have little effect on the amplification factor for all the breakwater configurations. The amplification factors at origin increase monotonically towards their asymptotic values. Also, Figs. 3(d)-3(f) show that a larger wave spreading angle clearly results in a smaller wave force except for the case of standing incident waves.

Many coastal and offshore structures are commonly designed with nonuniform porosity along the circumferential direction. Fig. 4 shows the wave amplification factor at the origin and wave forces $v s$. opening area angle for breakwaters with a partial solid wall (left) and a partial opening (right) located at $\theta=180^{\circ}$, and the porosity of the remaining part at $G_{0}=1$. For the breakwater with a partial solid wall (Fig. 4(a)) the amplification factors at origin generally decrease monotonically as the angle of the solid area increases with largest value for a standing wave, and reaches zero at $\theta \approx 345^{\circ}$. For the case of partial opening, Fig. 4(c) shows that the amplification factor at origin initially increases to a peak at the opening area reaching approximately half of the circumference then decreasing to 1 with increasing opening area angle. A plane incident wave is clearly seen to produce the largest amplification factor, while a standing wave generates the smallest. It clearly indicates that more surface disturbance occurs within the interior for the opening area angle in the range of $180^{\circ} \sim 360^{\circ}$ depending on the short-crestedness of the incident waves.

As clearly shown in Figs. 4(b) and 4(d), a general trend of increasing wave forces with decreasing short-crestedness is observed for breakwaters with either a partial solid wall or an opening. Though fluctuating with the solid or opening area angles, larger wave forces occur for the breakwater with a partial solid wall than that with uniform porosity without the solid part, while the breakwater with an opening tends to experience smaller wave forces. The largest wave forces occur when the solid area angle varies in the neighbourhood of $180^{\circ}$ for a plane incident wave. As the short-crestedness increases, the wave force for the breakwater with a partially solid wall peaks for the breakwater with a larger proportion of solid wall. In contrast, for the breakwater with a partial opening, the largest wave force always occurs at zero opening area, i.e., the breakwater of uniform porosity without any opening.

Fig. 5 shows the variation of wave amplification factor at the origin and wave forces on the breakwater for the cases of breakwaters with a partial solid 

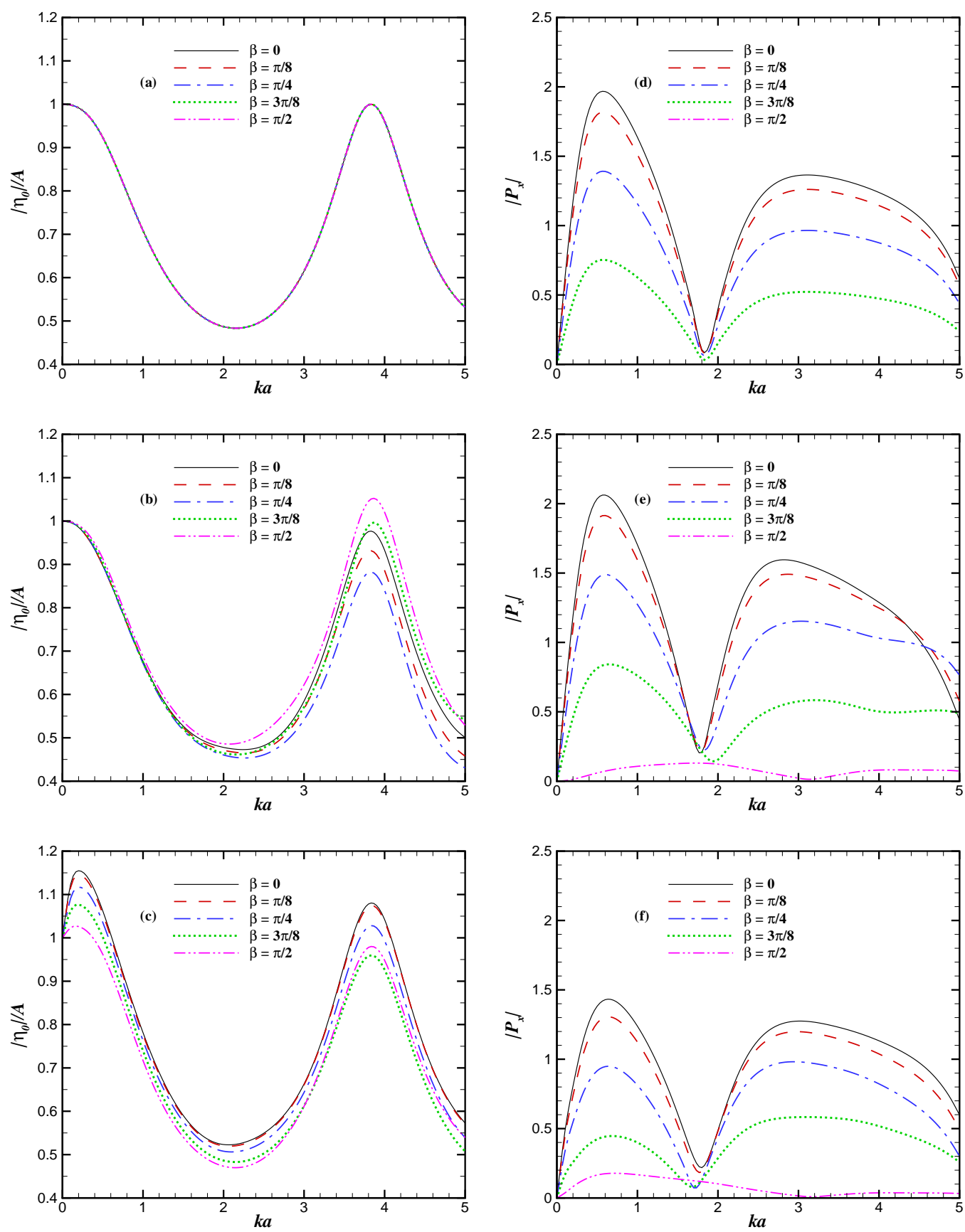

Figure 2. Variation of wave amplification factor at $r=0$ (left) and nondimensional wave force on the breakwater (right) with porosity $G_{0}=1 \mathrm{vs}$. $\mathrm{ka}$. 

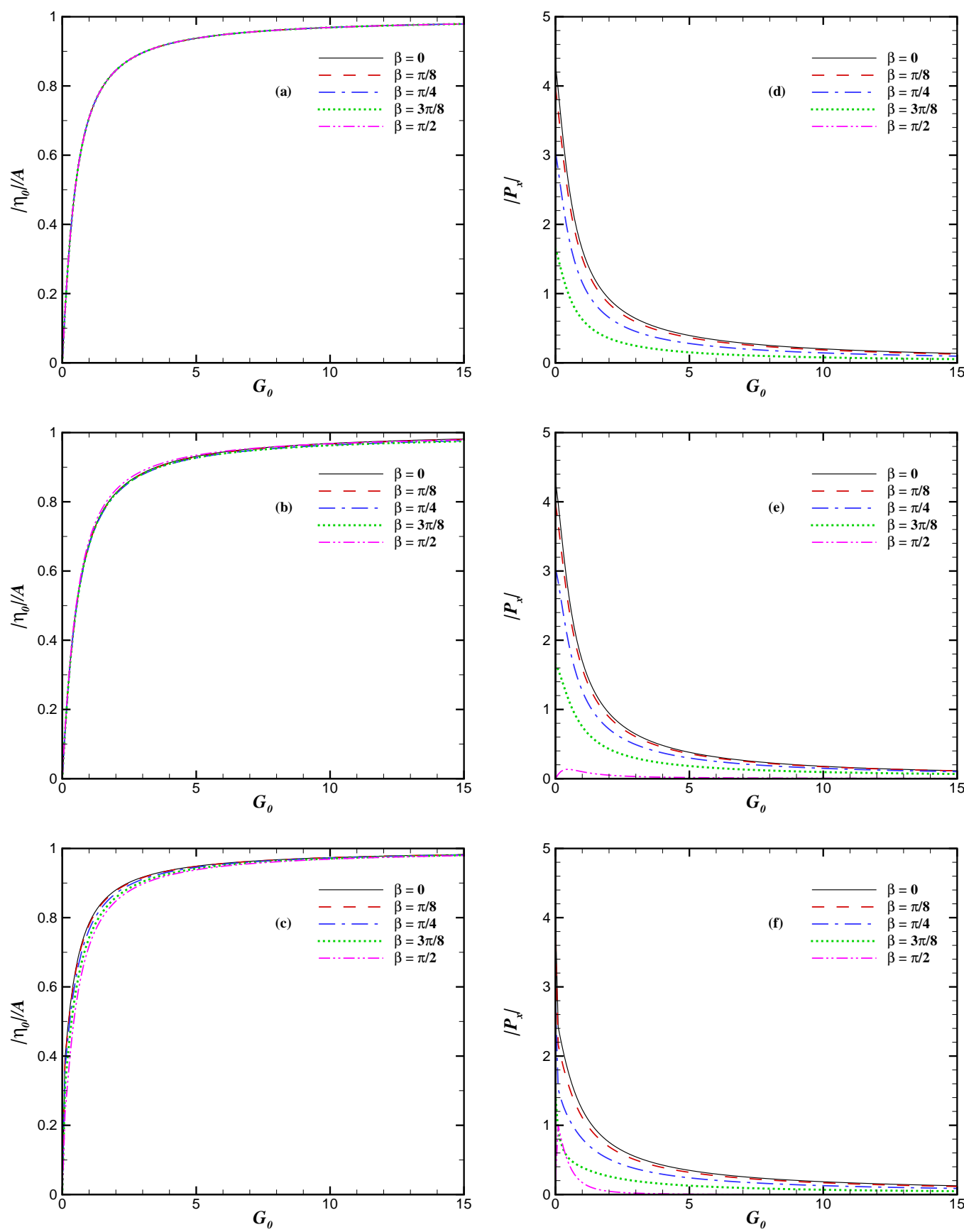

Figure 3. Variation of wave amplification factor at $r=0$ (left) and nondimensional wave force on the breakwater (right) at $k a=1 \mathrm{vs} . G_{0}$. 

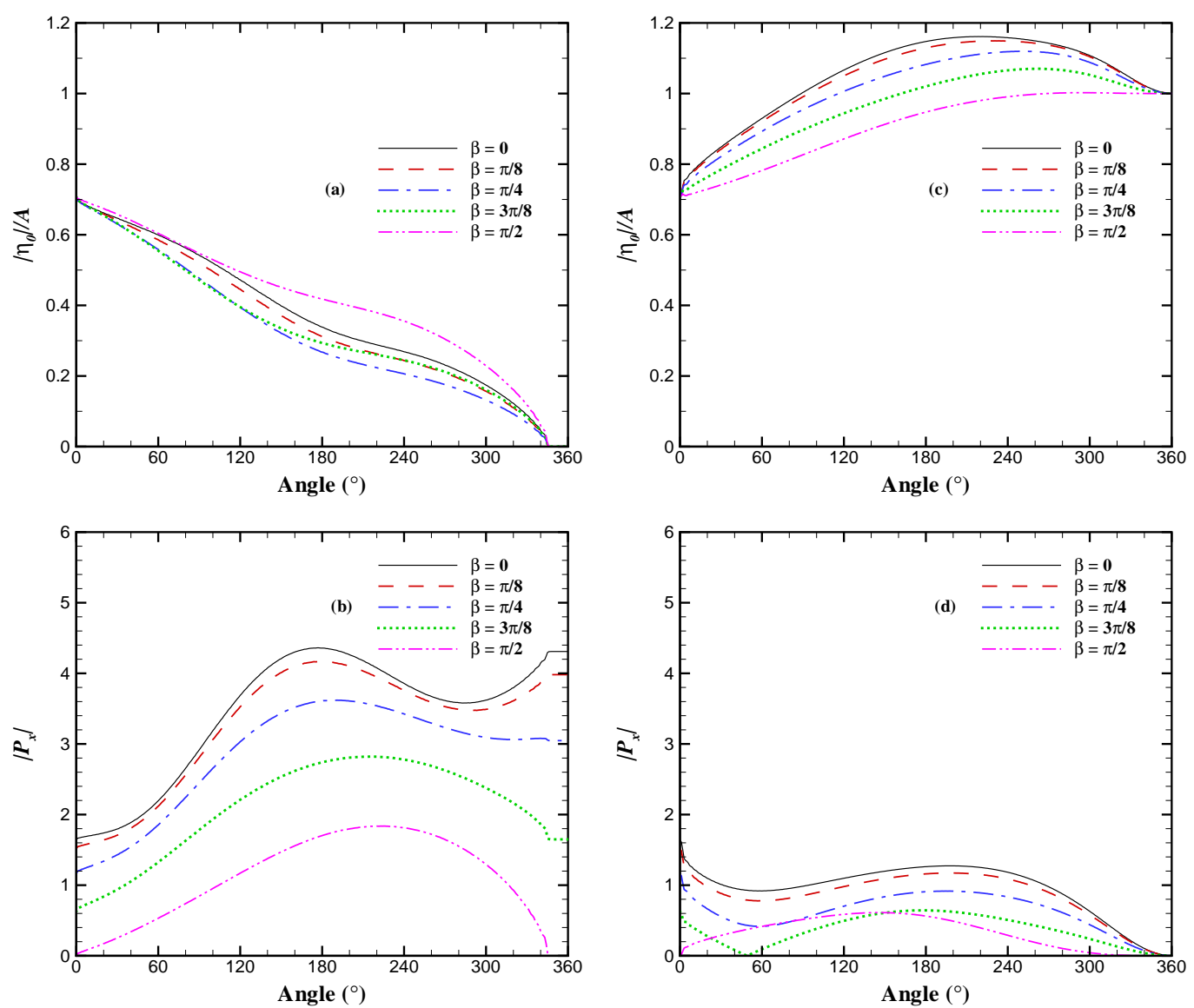

Figure 4. Variation of wave amplification factor at $r=0$ and nondimensional wave force on the breakwater with solid (left) or opening (right) centre at $\theta=180^{\circ}, k a=1$ and $G_{0}=1$ $v s$. solid or opening area angle.

wall (left) and a partial opening (right) $v s$. their centre location with a solid or an opening area angle of $10^{\circ}$ with the remaining part at $G_{0}=1$. As the location of the solid or opening centre varies, the amplification factor fluctuates whilst the largest amplification factor is often induced by either plane or standing incident waves. At some positions, different short-crestedness results in almost the same amplification factor (e.g., $70^{\circ}$ for breakwater with a solid wall, and $40^{\circ}$ and $120^{\circ}$ for breakwater with an opening). As for the wave forces in the $x$ direction, the earlier observations about smaller shortcrestedness and solid wall inducing larger wave forces still hold. However, the variation of the location of the solid or opening centre does not affect the magnitude of the inline force much. When the solid or opening centre are at $\theta=90^{\circ}$, the breakwater becomes symmetric along the $y$ axis leading to zero wave force in the $x$ direction due to standing waves. The wave force in the 
$y$ direction is rather small compared to its counterpart in the $x$ direction, and the largest wave force often occurs in either plane or standing incident waves.

Fig. 6 shows equi-amplitude (left) and equi-phase (right) contours for the interior region of the breakwater generated by incident plane, short-crested, and standing waves corresponding to the wave spreading angles $\beta=0, \pi / 4$ and $\pi / 2$ respectively. The breakwater has a partial opening at $175^{\circ}<\theta<$ $185^{\circ}$, and porosity of the remaining part is at $G_{0}=1$. Also wave number $k=1 \mathrm{~m}^{-1}$ and $a=5 \mathrm{~m}$. It can be seen that the wave patterns for shortcrested and standing incident waves are much more complex than the one for plane incident waves. The surface elevation within the breakwater is seen to decrease as $\beta$ increases. In addition to symmetry to the $x$-axis, wave elevation pattern due to a standing wave is seen almost symmetric to the $y$-axis as well. In this case, the slightly asymmetry to the $y$-axis is introduced by the small opening. The thick lines in phase contours represent changes from $\pi$ to $-\pi$. The amphidromic points, where equi-phase lines converge and the wave amplitude vanishes, clearly form for short-crested and standing incident waves. The phases near two adjacent amphidromic points rotate from $-\pi$ to $+\pi$ clockwise and counter-clockwise around the points respectively. For the standing incident wave component, the amplitudes in the transverse directions are small compared to their inline values, with a faster variation in the corresponding phase contours.

\section{Conclusions}

A general 3D short-crested wave interaction with a porous cylindrical breakwater is studied analytically by linear potential wave theory. Three basic configurations of the breakwater are investigated. The performance of the breakwater is examined by the effects of short-crested wave parameters, structural porosity, and the angle and position of the partial impermeable wall and opening on wave amplification factor, wave forces, and wave elevation contours. It is found that by making the porosity nonuniform, the amplification factor, wave forces, and elevation contours become more complex than its counterpart of uniform porosity. Incident waves with smaller short-crestedness along with solid walls generally result in larger wave forces, whilst an opening on the breakwater and limiting incident waves, i.e. plane or standing waves clearly lead to larger amplification factors within the breakwater. The effect of the location of the solid or opening centre appears to be insignificant on the inline wave force $\left(P_{x}\right)$, but rather significant on the transverse wave force $\left(P_{y}\right)$. However, since $P_{y}$ is one order smaller than $P_{x}$, we can conclude that the wave force is insensitive to the location of the solid or opening centre. Due to asymmetrical geometry, wave forces induced by standing incident waves 

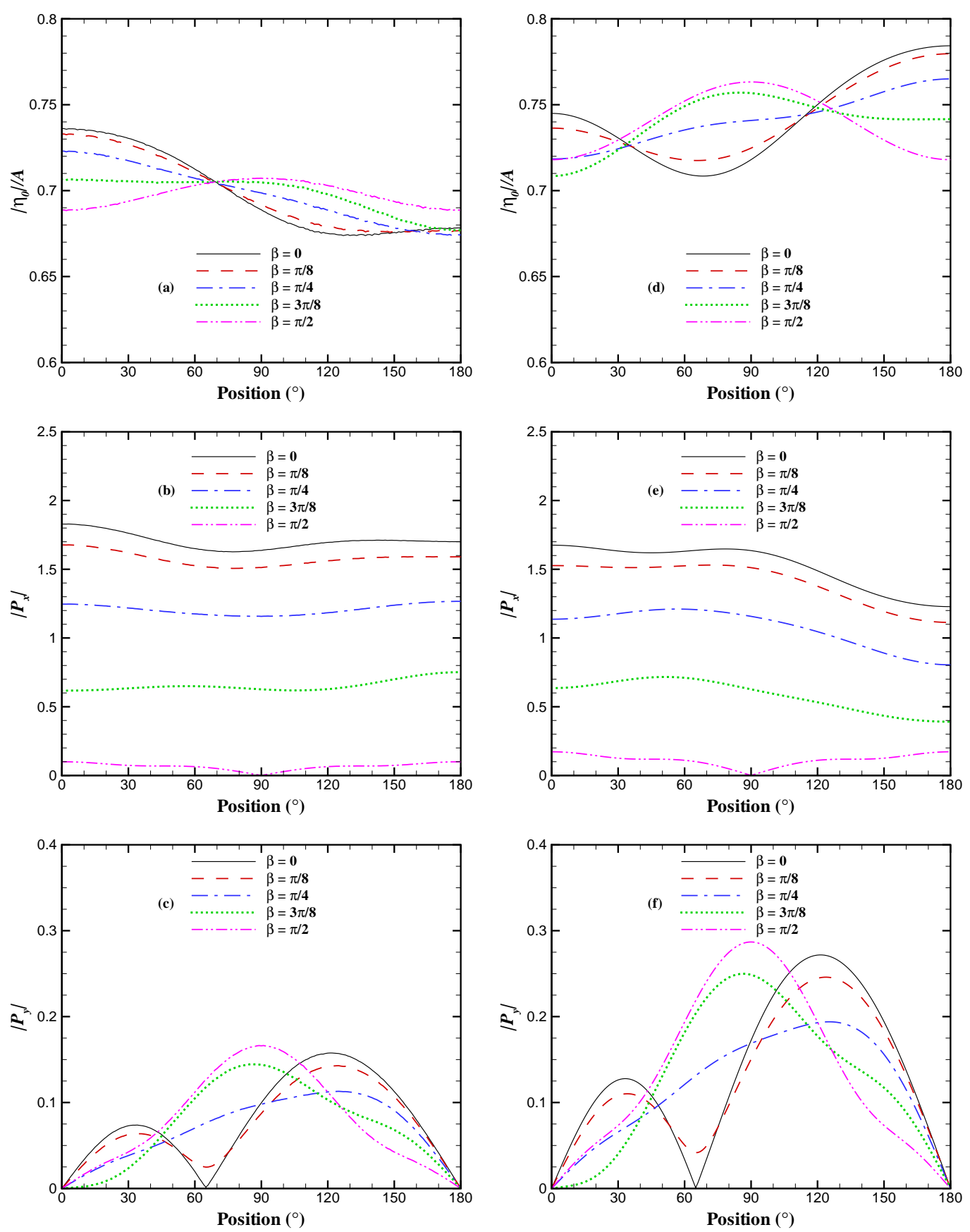

Figure 5. Variation of wave amplification factor at $r=0$ and nondimensional wave force on the breakwater with solid (left) or opening (right) area angle $10^{\circ}$ at $k a=1$ and $G_{0}=1 \mathrm{vs}$. the location of the solid or opening centre. 

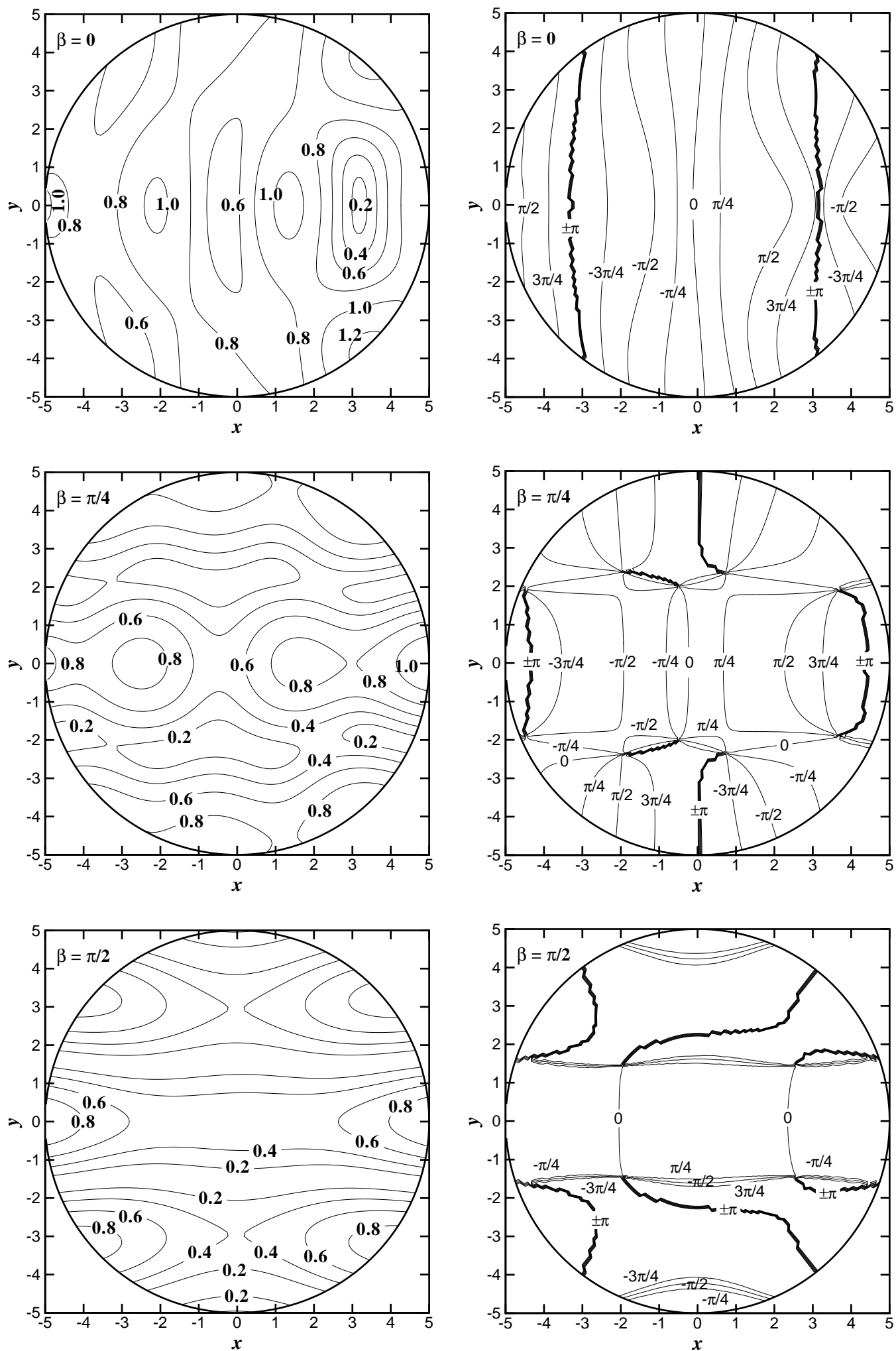

Figure 6. Equi-amplitude (left) and Equi-phase (right) contours for incident short-crested wave with short-crestedness angles $\beta=0, \pi / 4, \pi / 2$ and $k=1 \mathrm{~m}^{-1}$, and breakwater with partial opening at $175^{\circ}<\theta<185^{\circ}, a=5 \mathrm{~m}$, and porosity $G_{0}=1$. 
are no longer zero. Here the component in the direction perpendicular to the incident wave may come forth, though the magnitude is normally small.

It is hoped that the analysis presented and the results of the parametric study in the paper will be found useful in the design of coastal and offshore structures. They should be useful in selecting a suitable circular breakwater for a particular application.

\section{Acknowledgment}

This paper is based on the project funded by Australian Research Council (ARC) under Discovery Project Grant No. DP0450906. Authors would like to thank ARC for their financial support. The second author is grateful for the postdoctoral fellowship from Griffith University to support this research.

1. Chwang AT, Chan AT (1998) Interaction between porous media and wave motion. Ann R Fluid 30:53-84

2. Jarlan GE (1961) A perforated vertical wall breakwater - an examination of mass-transport effects in gravitational waves. The Dock \& Harbour Authority 41:394-398

3. Dalrymple RA, Losada MA, Martin PA (1991) Reflection and transmission from porous structures under oblique wave attack. J Fluid Mech 224:625-644

4. Huang LH, Chao HI (1992) Reflection and transmission of water wave by porous breakwater. J Waterw P 118(5):437-452

5. Chwang AT (1983) A porous wavemaker theory. J Fluid Mech 132:395-406

6. Yu X, Chwang AT (1994) Wave-induced oscillation in harbor with porous breakwaters. J Waterw P 120(2): 125-144

7. Yu X, Chwang AT (1994) Wave motion through porous structures. J Eng Mec 120(5):9891008

8. Wang KH, Ren X (1993) Water waves on flexible and porous breakwater. J Eng Mec 119(5):1025-1047

9. Tsai CP, Jeng DS, Hsu JRC (1994) Computations of the almost highest short-crested waves in deep water. Appl Ocean 16(6):317-326

10. Zhu S (1993) Diffraction of short-crested waves around a circular cylinder. Ocean Eng 20(4):389-407

11. Zhu S, Moule G (1994) Numerical calculation of forces induced by short-crested waves on a vertical cylinder of arbitrary cross-section. Ocean Eng 21(7):645-662

12. Zhu S, Satravaha P (1995) Second-order wave diffraction forces on a vertical circular cylinder due to short-crested waves. Ocean Eng 22(2):135-189

13. Fuchs RA (1952) On the theory of short-crested oscillatory waves. In: Gravity Waves, National Bureau of Standards Circular No. 521, Department of Commerce, USA. 187-200.

14. Taylor G (1956) Fluid flow in regions bounded by porous surfaces. P Roy Soc A 234:456-475

15. Li Y, Liu Y, Teng B (2006) Porous effect parameter of thin permeable plate. Coast Eng J 48(4):309-336 
